Historia y comunicación social

ISSN-e 1988-3056

https://dx.doi.org/10.5209/hics.66292

\title{
Fake News y difusión en Twitter: el caso de Curro, el perro "condenado"
}

\author{
Fátima Vila Márquez'; Sergio Arce García
}

Recibido: 6 de marzo de 2019 / Aceptado: 26 de septiembre de 2019.

Resumen. Animado por la revolución tecnológica, política, social y periodística de la web 2.0, el fenómeno de las noticias falsas ha irrumpido en la agenda mediática de la mano de la victoria de Trump en Estados Unidos y la polémica campaña del Brexit en Reino Unido. Largamente discutido por sus efectos en el panorama político y electoral, el espectro de las fakes news va mucho más allá de las campañas de propaganda y está promovido, además, por los cambios en el consumo y la distribución de las noticias y, sobre todo, en la monetización digital. Este artículo analiza, con el apoyo de técnicas de big data, la diseminación a través de Twitter de una noticia falsa de corte social --el caso de Curro, el perro "condenado"-, así como el comportamiento de los principales perfiles que la compartieron durante la semana de Navidad de 2018 con el fin de observar cómo se difunde y viraliza una noticia falsa, incluso de forma artificial. La investigación ahonda también en el comportamiento de la comunidad digital y de los contenidos con el fin de identificar dinámicas de coordinación y conductas relacionadas con los marcos de referencia y prejuicios preexistentes.

Palabras clave: Noticias falsas; redes sociales; bots; publicidad digital.

\section{[en] Fake News spreading on Twitter: Curro: The "condemned dog"}

\begin{abstract}
Spurred by the technological, political, social and journalistic revolutions related to Web 2.0, the phenomenon of Fake News has burst onto the media scene thanks to Trump's victory in the United States and the polemic Brexit campaign in the United Kingdom. Long discussed with regard to its effects on the political and electoral landscapes, the spectrum of Fake News extends far beyond propaganda campaigns and is driven as well by changes in the consumption and distribution of news and, above all, by changes in digital monetization. Using Big Data techniques, this article analyses the case of the dissemination of a false social media news story via Twitter: the case of Curro, the "condemned" dog. The behavior of the principal actors who shared the story during the week of Christmas, 2018, is also analysed, with the aim of observing how a news item is spread and viralised, even if by artificial means. The research also delves into content sharing and digital community behaviors in order to identify coordination dynamics and conduct related to pre-existing prejudices and frames of reference.
\end{abstract} Keywords: Fake news, social media, bots, digital advertising

Sumario: 1. Introducción; 1.1. Las fake news y el nuevo panorama publicitario; 1.2. De los legacy media a las redes sociales: la disolución del emisor de información; 1.3. Curro, el perro "condenado"; 2. Metodología; 2.1. Captura de mensajes; 3. Resultados; 3.1. Cronología de elementos encontrados; 3.2. Análisis de redes; 3.3. Utilización de bots en la difusión; 3.4. Análisis de sentimientos; 3.5. Minería de texto de los discursos vertidos; 4. Conclusiones; 5. Bibliografía.

\footnotetext{
1 Universitat de Barcelona. fatimavila@ub.edu

2 Universidad Internacional de La Rioja (UNIR). sergio.arce@unir.net
} 
Cómo citar: Vila Márquez, F.; Arce Sergio, S. (2019). Fake News y difusión en Twitter: el caso de Curro, el perro "condenado", en Historia y comunicación social 24 (2), 485-503.

\section{Introducción}

El término noticia ha recibido numerosas aproximaciones en el marco de los estudios periodísticos y sociales a partir de mediados del siglo XX. Entendida como relato o "reporte de un suceso reciente, interesante y significante" (Kershner, 2005) y generalizada como un producto del Periodismo, se espera de ella que sea "información independiente, confiable, precisa y comprensible" (Kovach y Rosenstiel 2007:11). Como constructo social, el papel subjetivo de los periodistas en la elaboración de noticias ha sido largamente discutido (Herman y Chomsky, 2002; Tuchman, 1978), así como la responsabilidad de otras fuerzas mediadoras como gobiernos, audiencias y anunciantes en su configuración (Shoemaker y Reese, 2013). Esta mediación comúnmente aceptada no desmerece, sin embargo, que el concepto de noticia vaya, intrínseca y conceptualmente, unido al de verdad, haciendo que conceptos como las fake news planteen un oxímoron (Tandoc, Lim y Ling, 2017) vinculado a contextos de desinformación espontáneos o intencionados (Wardle, 2017).

El término fake news adquirió una gran importancia en el marco de las elecciones presidenciales de EEUU en 2016 para hacer referencia al enorme flujo de desinformación digital (Wardle, 2017) que rodeó la victoria del republicano Donald Trump. Lo mismo ocurrió con el referéndum de salida del Reino Unido de la UE, en el mismo año. Hoy por hoy, sin embargo, el estudio del fenómeno es aún un campo joven y en crecimiento que abarca una enorme variedad de contenidos online con propósitos y naturalezas diversos. Así, junto a ejemplos de propaganda política gris o negra, es posible encontrar fake news en la prensa satírica y expresamente falsa. En su revisión de la bibliografía sobre el tema desde 2003 a 2017, Tandoc, Lim y Ling (2017) elaboran una tipología de fake news basada en dos variables: el nivel de autenticidad (facticity) y de engaño (deception). Así, estos autores señalan seis tipologías: Noticias Satíricas, Parodias, Noticias Fabricadas, Manipulación Fotográfica, Publicidad y RRPP, y Propaganda. Para Allcott y Gentzkow (2017) una noticia es una fake new cuando responde a "artículos noticiosos intencionada y verificablemente falsos capaces de engañar a los lectores". Siguiendo la aportación de estos autores, se considerarían fake news propiamente dichas las Noticias Fabricadas y la Propaganda. Ambos autores apuntan que existen dos motivaciones básicas tras el fenómeno: ideológicas o económicas. En el primer caso, los productores de noticias falsas promocionan determinadas ideas o personas o, en ocasiones, las desacreditan, en el marco de procesos propagandísticos. En el otro lado, están las historias extravagantes y falsas que despiertan la curiosidad de la comunidad digital y proveen de clics a los portales convirtiendo esas visitas en beneficios gracias a la publicidad. La importancia de esta última motivación es tal que, como señalan Bakir y McStay (2017:13), "muchos de los sitios de noticias falsas creados en el marco de las elecciones de 2016 se crearon no para hacer propaganda, sino dinero". 


\subsection{Las fake news y el nuevo panorama publicitario}

La dependencia económica de los medios de comunicación de los ingresos por publicidad es un rasgo intrínseco a la prensa moderna. La irrupción de nuevas formas de tarificación digital ha transformado las reglas del juego haciendo que la contratación directa medio/anunciante ceda ante formas de publicidad programática que, servidas de decisiones algorítmicas y segmentando por historial y preferencias de usuario -y no por medio-, diluyen el control que se tenía sobre el lugar donde aparecían los anuncios. La subasta programática (advertising exchange) no solo elimina el esfuerzo de vender los espacios publicitarios sino que también reduce los costes, lo que ha impulsado su auge en los últimos años (Peterson, 2018) así como la proliferación de medios de baja calidad que obtienen ingresos a través de este medio.

\subsection{De los legacy media a las redes sociales: la disolución del emisor de información}

A esta importante transformación en el ámbito publicitario hay que añadir un importante cambio estructural que tiene que ver con la traslación del poder informativo desde los legacy media (televisión y prensa) del siglo XX al entorno digital y social del siglo XXI. Los medios tradicionales, si bien continúan teniendo un papel importante como productores de noticias, han perdido su poder como distribuidores o gatekeepers, tareas en las que toman una importancia inusitada los motores de búsqueda, las redes sociales o las aplicaciones de mensajería (Bell, 2017; Nielsen y Ganter, 2017). La digitalización, además, ha supuesto un gran desafío para la forma en la que tradicionalmente se entendían las noticias abriendo la posibilidad de que los no periodistas puedan alcanzar con sus contenidos a una audiencia masiva. Primero con los blogs y luego con las redes sociales, los usuarios pueden publicar información, fotos y vídeos sobre noticias de interés de las que pueden ser testigos de primera mano (Hermida, 2011; Jewitt, 2009) y, gracias a la particular configuración de estas redes, la información se viraliza rápidamente. Esta revolución en la producción de contenidos ha significado también una dispersión del tradicional concepto de fuente que, al alejarse del emisor de origen, es recibida cada vez más a través de un conjunto de capas con varios niveles de proximidad con el lector (Kang et al., 2011:721) requiriendo un esfuerzo para su correcta identificación que el lector no siempre está dispuesto a realizar. A esto se suma que cada post está acompañado de rankings de popularidad (Sundar, 2008). Variables como los Me Gusta, los retuits o comentarios aumentan el alcance de un contenido incrementando sus posibilidades de ser visto por otras personas y, de esta forma, seguir creciendo en un círculo que se retroalimenta y es perfecto para la diseminación de datos no contrastados. Finalmente, como se ha demostrado al analizar el comportamiento y desarrollo de los bots de noticias (Lokot y Diakopoulos, 2016), los programas informáticos desarrollados por las propias redes automatizan este círculo vicioso al ordenar la ingente cantidad de contenidos para que los consumidores reciban información de manera selectiva (Iyengar y Hahn, 2009:34). Como consecuencia, los contenidos más virales aumentan exponencialmente y los lectores menos formados interpretan su éxito como un elemento de legitimidad.

Esto adquiere aún más importancia si tenemos en cuenta el papel que los lectores dan a las redes sociales en su proceso de información. En EEUU, un $62 \%$ de los 
adultos declaraban en 2016 que usaban las redes sociales para informarse y un 66\% que lo hacía a través de Facebook (Gottfried y Shearer, 2016). Según El libro blanco de la información (2017) publicado por la Asociación de Medios de Información (AMI), el 56,8 \% de los españoles consumen noticias a través de las redes. Según esta investigación, Facebook, Twitter e Instagram se revelan como la principal forma de consumir información, no ya a través de los perfiles de los medios de comunicación tradicionales, sino a través de contenidos compartidos por otros usuarios. El informe arroja también otro dato significativo en cuanto a la profundidad de ese hábito de consumo, y es que más de la mitad de los lectores, el 57,8 \%, solamente leen los titulares y no hacen clic en el enlace para conocer el contenido completo del artículo. Se trata, por tanto, de un consumo intermediado y fragmentado, perfecto como caldo de cultivo para la proliferación de noticias falsas.

Otra de las cuestiones a tener en cuenta al analizar el fenómeno de asimilación de las fakes news es la tendencia de los usuarios no familiarizados con los medios a tomar como ciertos aquellos portales que se asemejan en forma a los medios de comunicación tradicionales, tal y como demostraron Flanagin y Metzger (2007). Un fenómeno que es aún más peligroso si se tiene en cuenta cómo las fakes news imitan "la omnipresencia de las noticias reales construyendo una red de sitios falsos" (Tandoc, Lim y Ling, 2018). Como señala Fernández-García (2017), esta falta de una alfabetización mediática ahonda además en dos de los fenómenos que más interés suscitan respecto al consumo de noticias: el filtro burbuja (filter bubble), que "obstaculiza el acceso a la información que podría desafiar o ampliar nuestra visión del mundo", y "las cámaras de resonancia (echo chambers), en las que la información, las ideas o creencias son amplificadas por transmisión y repetición en un sistema cerrado donde las visiones diferentes se descartan o se representan de forma minoritaria".

\subsection{Curro, el perro "condenado"}

La siguiente investigación analiza el proceso de difusión y diseminación de una de las noticias falsas distribuidas en el ecosistema social español, en la Navidad de 2018: el caso de Curro, el perro falsamente ordenado a "sacrificar" por parte de un Tribunal de Huelva tras morder al ladrón -de procedencia rumana y con numerosos antecedentes- que amenazaba a su dueño. La noticia fue distribuida primero desde varios portales claramente identificados como emisores de noticias falsas (Peinado, 2018; Ramírez y Castellón, 2018), como CasoAislado.es, y viralizada más tarde por perfiles de Twitter que multiplicaron y dieron apariencia de legitimidad a la noticia. Como se verá a continuación, es posible identificar entre estos perfiles tanto usuarios reales como un gran porcentaje de posibles bots. Por la especial sensibilidad hacia el tema, el caso de Curro no solo registró varios miles de tuits, sino que animó varios hashtags (\#CurroEstaEnMiCasa, \#NoAlSacrificiodeCurroenHuelva) e inspiró varias campañas a través del portal Change.org hasta su total desmontaje.

Considerada como una plataforma perfecta para la rápida diseminación de noticias de última hora y analizada largamente como una red fundamental en los procesos de periodismo participativo (Hermida, 2011), Twitter no solo ha cambiado la forma de distribuir la información de actualidad sino que ha modificado en sí mismo el concepto de noticia logrando que un texto de 140 caracteres -ahora 280- pueda ser considerado como tal, si proviene de una fuente acreditada (Tandoc, Lim y Ling, 
2017). En el caso que nos ocupa, la especial naturaleza de esta red sirvió para viralizar en dos picos de tiempo la difusión del caso.

Siguiendo la tipología de fake news elaborada por Tandoc, Lim y Ling (2017), el caso que ocupa se enmarcaría en el supuesto "Fabricación de Noticias", esto es, información intencionadamente falsa publicada bajo la apariencia formal de una noticia para crear legitimidad. Al contrario de la parodia, las noticias fabricadas o, más explícitamente, las mentiras, no parten de un pacto tácito entre el medio de comunicación y el lector, sino todo lo contrario. El autor de la noticia quiere intencionadamente confundir al que lee. La problemática se hace más compleja cuando determinados sectores asumen como verídica la mentira por razones partidistas y comienzan a diseminarla dotándola de legitimación externa. En estos casos, las noticias fabricadas se entretejen en una narrativa o frame preexistente, parten a menudo de un sesgo político que el lector acepta como legítimo y se instalan con mayor facilidad cuando existe una tensión social -política, sectaria, racial o cultural- detrás. La voluntad de conseguir ese efecto es evidente en el momento en el que las primeras distribuciones de tuits sobre Curro mencionan la cuenta oficial del partido animalista PACMA -@PartidoPACMA- y a algunas personalidades comprometidas con los derechos de los animales.

Es importante también señalar aquí el rol de los perfiles y medios encargados de “desmontar" la falsa historia. Como señala Magallón (2018), el auge de las fakes news ha traído consigo la puesta en marcha de diversos sistemas de verificación periodísticos, académicos y gubernamentales. En el caso que ocupa, esa tarea de fact-checking es llevada a cabo desde la prensa online y también desde Maldito Bulo, un proyecto periodístico que formó parte del grupo de alto nivel sobre fake news de la Comisión Europea en 2018 y que desde hace años colabora desmontando bulos en televisión (La Sexta), radio (RNE y RAC1), diarios digitales (eldiario. es) y su propia web (Maldita.es ${ }^{3}$ ). Junto a esta plataforma, el otro canal verificador fue el propio Twitter, como lugar donde se difunden y corrigen fragmentos de información (y desinformación) en un proceso interactivo que involucra a periodistas profesionales y usuarios no profesionales (Coddington, Molyneux y Lawrence, 2014).

\section{Metodología}

\subsection{Captura de mensajes}

Para poder capturar los tuits sobre Curro, esta investigación utilizó el software R, a través de su entorno visual RStudio en su versión 1.1.456. Con él, y la ayuda de librerías específicas, se llevó a cabo una conexión a la interfaz de programación de aplicaciones (API) de Twitter para descargar todos los tuits que contuvieran, al menos, dos de las siguientes palabras: "perro", "justicia", "curro" y "alano" durante el periodo del 20 de diciembre de 2018 al 1 de enero de 2019, ambos incluidos.

Asociación Maldita contra la Desinformación Periodismo Educación Investigación y datos en nuevos formatos (2019). Maldita.es. Disponible en: https://maldita.es/ [Consulta: 4 de marzo 2019] 
Tras la recogida de datos se les aplicaron diversas técnicas de análisis de Big Data:

- Análisis de redes: aplicación de algoritmos matemáticos para determinar a los autores más seguidos o influencers. Se empleó el programa informático Gephi, en su versión 0.9.2, para realizar un análisis gráfico de redes y conexiones entre usuarios. Se estudió así la relación entre datos a través de una representación gráfica de nodos (usuarios) y sus conexiones o enlaces, en función de la distancia entre puntos, tamaño y grosor de las líneas que los unen (Bastian et al., 2009). Se aplicaron sucesivamente algoritmos para determinar cada posicionamiento y se asignó mayor tamaño a los círculos y textos en función de su relevancia.

- Análisis de emociones: cada vez más importante en los estudios de opinión pública, el análisis de las emociones es una disciplina que gana protagonismo (Fernández Vallejo, 2018) para identificar sentimientos básicos -alegría, tristeza, enfado, miedo, disgusto, sorpresa, confianza y anticipación o pensamiento racional- en diversos medios (Sautera et al., 2010; Plutchik, 1980). Con el fin de contrastar y asignar emociones a cada tuit, este estudio empleó el léxico de atribución desarrollado por el National Research Council of Canada versión 0.92 en su versión en castellano (Mohammad y Turney, 2010).

- Análisis de minería de texto: se procedió a un estudio masivo de los textos eliminando subjetividades en su clasificación para obtener, con el apoyo de técnicas estadísticas de framing, cuáles son los marcos o enfoques principales. Para conseguirlo, este trabajo utilizó el software KH-Coder en su versión 3.0 y aplicó la metodología tipo stemming, basada en el recorte de las palabras, para buscar sus variantes morfológicas y agruparlas (Arce y Menéndez, 2018). Para concretar los principales marcos se aplicó un análisis multidimensional bivariado a los ocho principales cluster o agrupaciones de palabras a través del método de varianza mínima de Ward (1963) eliminando las palabras vacías (stopwords) que no aportan significado: artículos, conjunciones y palabras de uso común o conexión entre términos según www.rank.n1 $1^{4}$.

\section{Resultados}

\subsection{Cronología de elementos encontrados}

El análisis de datos sobre el perro Curro en Twitter abarcó desde el día 20 de diciembre de 2018 hasta la madrugada del 2 de enero de 2019 y supuso la recogida de un total de 9.121 tuits.

Ranks (2019). Ranks NL. Disponible en: https://www.ranks.nl/stopwords/spanish [Consulta: 4 de marzo 2019] 


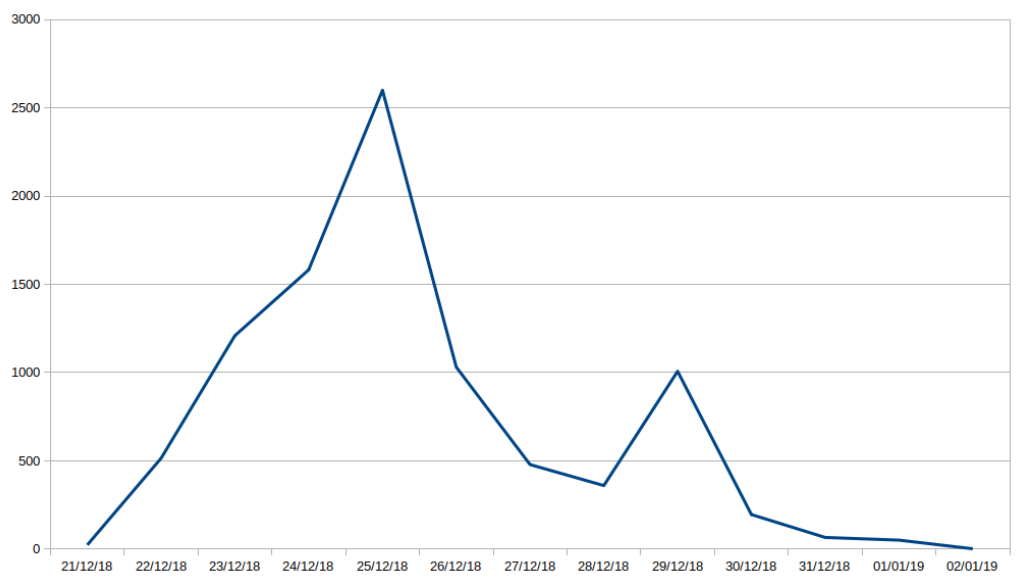

Figura 1: número de tuits por día. Fuente: Elaboración propia

Lanzada por la web club-caza.com el 20 de diciembre de 2018 a las 15:24, en las primeras horas la noticia de Curro no tiene eco en Twitter. El 21 de diciembre, sin embargo, la información aparece en los portales despiertainfo.com a las 13:34 horas y casoaislado.es. Este último portal, si bien no muestra la hora de publicación, es el primero en difundir un tuit a las 10:41 al que siguen, en el primer minuto, tres retuits, y otros diez más en los primeros diez minutos.

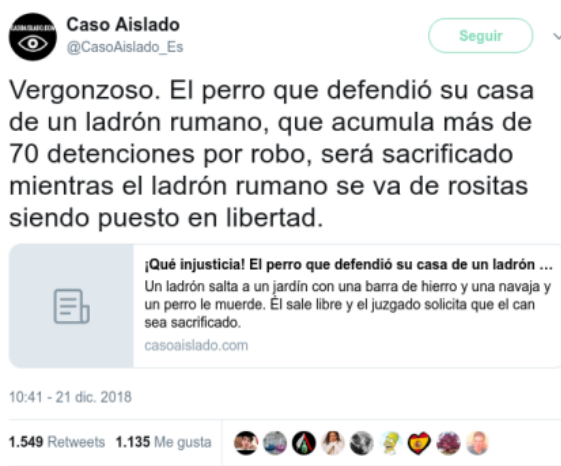

Figura 2: Primer tuit de casoaislado.es sobre Curro. Fuente: Twitter.

Los primeros tuits que no proceden de casoaislado.es aparecen, sin citar fuente, desde la cuenta@CarlosAsuajeS, propiedad de un activista opositor venezolano a las 10:49 y a las 10:55. En la difusión, se lanza el hashtag \#NoAlSacrificiodeCurroenHuelva y un tercer tuit interpelando a otros usuarios con miles de seguidores con el fin de conseguir sus retuits. A las 11:15, se difunde otro mensaje desde @ PortalAduanero, otra cuenta opositora venezolana que utiliza el mismo hashtag y ruega colaboración con retuits. 

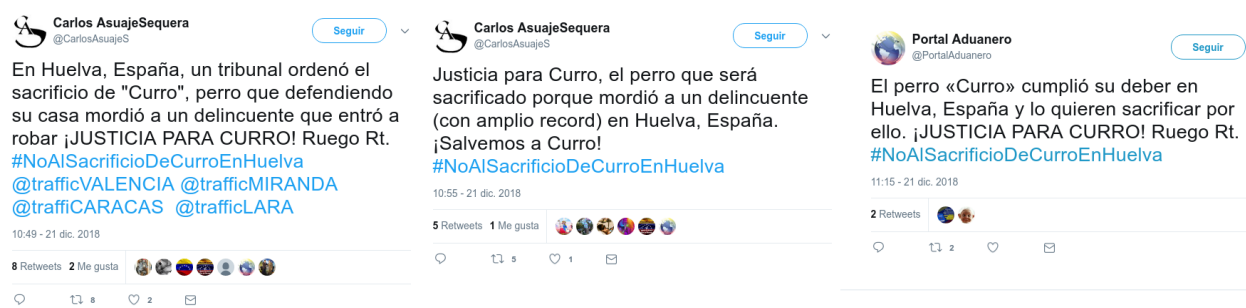

Figura 3: Primeros tuits sobre Curro encontrados en la red Twitter. Fuente: Twitter.

A partir de la difusión de las informaciones en despierta.info y casoaislado.es, algunos usuarios comienzan a escribir sobre Curro mencionando la cuenta oficial del partido animalista PACMA y a personalidades del mundo de la cultura y la sociedad. La difusión, con todo, es escasa el día 21 (solo 24 tuits) pero se incrementa hasta los 515 el día 22 de diciembre, gracias a numerosos reenvíos o retuits. Las llamadas a PACMA consiguen que, durante el mismo día 21, una activista cree una página en el portal Change.org pidiendo "No al sacrificio de Curro"5 a los juzgados de Huelva. Una petición que logró, en los dos meses siguientes y fundamentalmente desde Facebook y WhatsApp, más de 61.000 adhesiones. Es preciso añadir aquí que, unos días más tarde, la autora añade una nota aclaratoria pidiendo disculpas por difundir una noticia falsa. El día 22 surge otra petición en Change.org con comentarios similares a los que aparecen en casoaislado.es, consiguiendo, también, más de 30.000 adhesiones en los dos meses siguientes de una forma similar ${ }^{6}$.

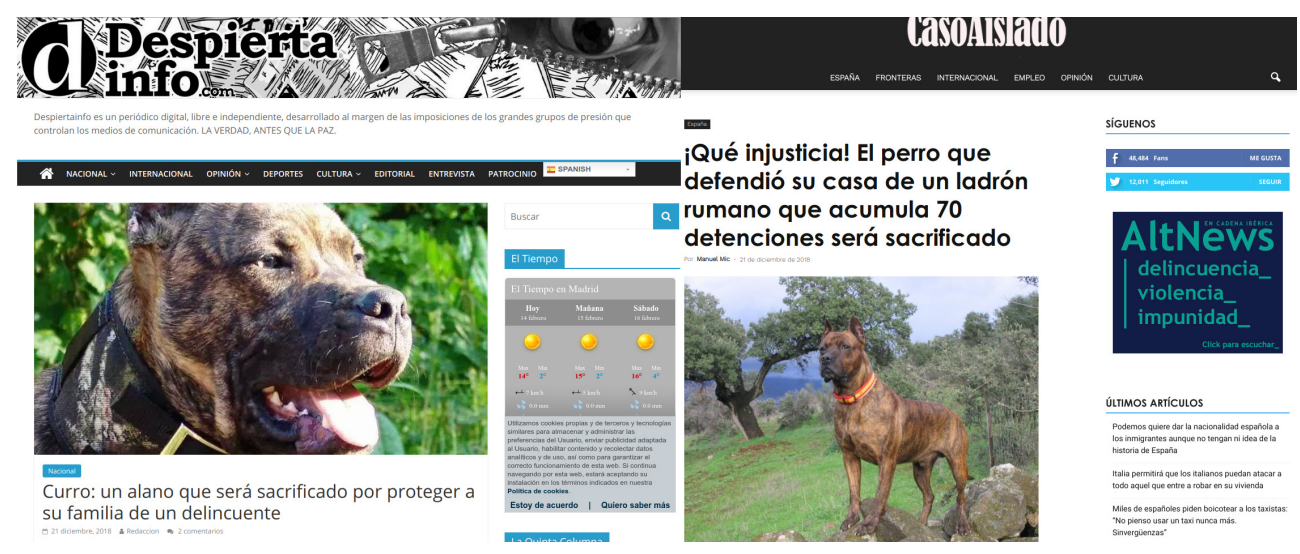

Figura 4: Portadas de webs del día 21 de diciembre de 2018. Fuentes: despierta.info y casoaislado.es

E1 día 23, Casoaislado.es publica otra información titulada "Miles de españoles salen en defensa de Curro, el perro que será sacrificado por defender su casa de un ladrón rumano"7, lo que multiplica el número de tuits relacionados con el caso hasta

5 Change.org, PBC (2018). Change.org. Disponible en: https://www.change.org/p/juzgado-de-huelva-no-al-sacrificio-del-perro-curro [Consulta: 4 de marzo 2019]

6 Change.org, PBC (2018). Change.org. Disponible en: https://www.change.org/p/todos-los-amantes-de-losanimales-y-perros-salvemos-a-curro-perro-alano-espa\%C3\%B1ol-42e0bc60-96f3-4e80-939c-7e8204c00231 [Consulta: 4 de marzo 2019]

7 Gallardo Sáinz, G. (2018). "Miles de españoles salen en defensa de Curro, el perro que será sacrificado por 
los 1.209. Es en este momento cuando aparece el primer tuit, del periodista@oriolguellipuig, que denuncia la falsedad de la noticia y la utilización interesada de los animalistas.

Durante el 24 de diciembre, hasta casi las 10:00 de la mañana se producen redifusiones de los seguidores animalistas con 188 tuits. A las 9:52, la cuenta oficial de Casoaislado.es cuenta con 32 retuits pero, en los siguientes 15 minutos, alcanza los 473 Me Gusta y 780 RT. Los tuits seguirán creciendo hasta los 1.582 sobre todo a partir de las 15:00 horas. Las cuentas de origen tienen en común la temática animalista, su autoidentificación como españoles y el apoyo a diversas causas, desde votantes del partido Vox a seguidores del Real Madrid, Real Valladolid, Sevilla o Betis. Estas cuentas viralizan y lanzan nuevos mensajes alrededor de la noticia gracias al apoyo de otros seguidores hasta alcanzar más de 500 redifusiones. Un usuario animalista (@adoptaunavidaMD) también es retuiteado extensamente pero no llega a alcanzar las difusiones que logra casoaislado.es.

El día de Navidad la noticia alcanza los 2.599 tuits, muchos de ellos retuits, y se convierte en trending topic del día. Es a las 8:00 cuando aparece por primera vez el hashtag \#CurroEstaEnMiCasa, a través de una seguidora animalista que cita como fuente a Casoaislado.es e interpela en varias ocasiones a la cuenta del Ministerio del Interior para detener el sacrificio. Dicho hashtag será redifundido por seguidores animalistas y por seguidores de Vox. A las 7:16 del día 25 la presidenta del partido PACMA advierte que la noticia podría ser falsa, aunque su tuit no se mueve hasta el final del día ya que los tuits originales sobre Curro y la petición al juzgado están en su mayor auge. Es entonces cuando la web club-caza.com publica una supuesta entrevista con el dueño de Curro para corroborar su historia y reanimar el debate ${ }^{8}$.

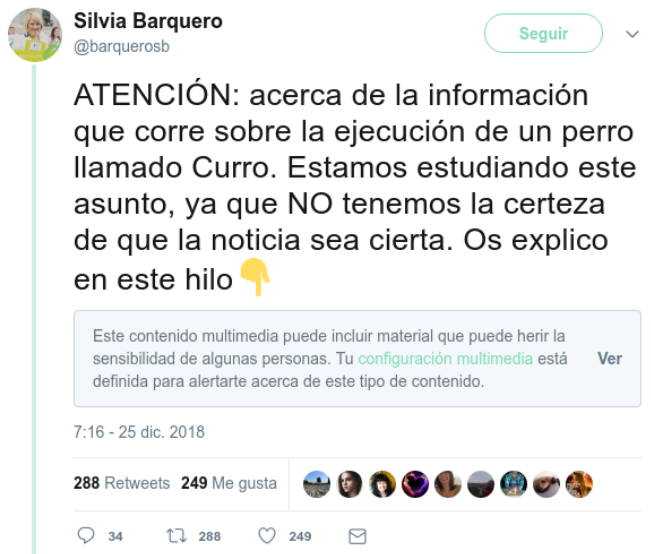

Figura 5: Tuit de la presidenta de PACMA advirtiendo sobre la veracidad de la información, del día 25 de diciembre de 2018 a las 7:16. Fuente: Twitter.

defender su casa de un ladrón rumano". Caso Aislado, 23 de diciembre de 2018. Disponible en: https://casoaislado.com/miles-de-espanoles-salen-en-defensa-de-curro-el-perro-que-sera-sacrificado-por-defender-su-casa-de-un-ladron-rumano/ [Consulta: 4 de marzo 2019]

8 CdC (2018). "Última hora sobre el caso del alano español condenado al sacrificio por defender su casa". Web de Caza, 25 de diciembre de 2018. Disponible en: https://www.club-caza.com/actualidad/actualver.asp?nn=8247 [Consulta: 4 de marzo 2019] 
El día 26 un artículo del periodista@GerardoTC en la revista online CTXT -"Curro no está en tu casa, está el racismo" - denuncia la falsedad y empieza a recibir atención social. Diversos mensajes muestran la manipulación de las fotos sacadas de una web para aficionados a los perros ${ }^{10} \mathrm{y}$ el número de tuits desciende a 1.031. Es entonces cuando casoaislado.es publica, basándose en la entrevista aparecida en club-caza.com, una corroboración de la información con el titular "El ladrón rumano al que Curro mordió al defender su vivienda, denunciará a sus dueños por daños y perjuicios" haciéndose eco, incluso, de las informaciones que denuncian que es un bulo $^{11}$. Es en este momento cuando, desde su perfil en la red, la investigadora Mariluz Congosto (@congosto), de la Universidad Carlos III de Madrid, publica una serie de comentarios alertando sobre el caso, así como sobre la participación de cuentas presumiblemente falsas.

El día 27 los tuits comienzan a descender. Se contabilizan 479 ese día y 360 un día después, la mayoría relacionados con la cuenta @Mazzinguerzett1 desde la que se difunde la noticia original. El día 29, otra web sensacionalista, Periodistadigital. com, se hace eco del caso de Curro y el ladrón rumano ${ }^{12}$. Es entonces cuando Alfredo Pascual, de El Confidencial, lanza un artículo desmontando la falsedad ${ }^{13}$. A partir de esta aparición el número de tuits vuelve a ascender a 1.007 en el día y responden, en su mayoría, a denuncias sobre la falsedad del caso. A partir del día 30 , los impactos bajan a 196 tuits, a 66 el 31 y a 51 el día 1, coincidiendo con la denuncia del caso en Maldito Bulo.

\subsection{Análisis de redes}

El análisis de los tuits recogidos arroja 1.309 tuits directos (14,35\%) y 7.812 retuits (85,65\%), mostrando así que se trató de un fenómeno de redifusión más que de creación de contenidos por los propios usuarios. El estudio del gráfico de redes, que da mayor importancia a los usuarios con vector medio o eigenvector superior y determina aquellas cuentas que son más influyentes (influencers), muestra que solo hay unos pocos creadores de opinión. A través de un análisis de estructuras de redes en los retuits con Gephi 0.9.2 se determinó, en un análisis de cluster, una modularidad (Blondel et al., 2008) de 0,804 . Un dato muy alto que permite determinar grupos bien definidos con asociaciones propias.

9 Tecé, Gerardo (2018). “Curro no está en tu casa, está el racismo”. Revista Contexto, 28 de diciembre de 2018 . Disponible en: https:/ctxt.es/es/20181226/Firmas/23655/Gerardo-Tece-curro-el-perro-espa\%C3\%B1ol-racismo-inmigrantes.htm [Consulta: 4 de marzo 2019]

10 Leonardo Bernardo Hernández (2018). Eperros.com. Disponible en: https://eperros.com/alano-espanol [Consulta: 4 de marzo 2019]

11 Gallardo Sáinz, G. (2018). "El ladrón rumano al que Curro mordió al defender su vivienda, denunciará a sus dueños por daños y perjuicios". Caso Aislado, 26 de diciembre de 2018. Disponible en: https://casoaislado. com/el-ladron-rumano-al-que-curro-mordio-al-defender-su-vivienda-denunciara-a-sus-duenos-por-danos-yprejuicios/ [Consulta: 4 de marzo 2019]

12 Periodista Digital (2018). "Este es el perro fiel y valiente que "será sacrificado por morder a un ladrón rumano". Periodista Digital, 29 de diciembre de 2018. Disponible en: https:/www.periodistadigital.com/ciencia/mundo-animal/2018/12/29/este-es-el-perro-fiel-y-valiente-que-sera-sacrificado-por-morder-a-un-ladron-rumano. shtml [Consulta: 4 de marzo 2019]

13 Pascual, Alfredo (2018). La verdadera historia del perro que "será sacrificado por morder a un ladrón rumano". El Confidencial, 29 de diciembre de 2018. Disponible en: https://www.elconfidencial.com/espana/2018-12-29/ curro-alano-espanol-rumano-huelva-sacrificio-vox_1732106/ [Consulta: 4 de marzo 2019] 


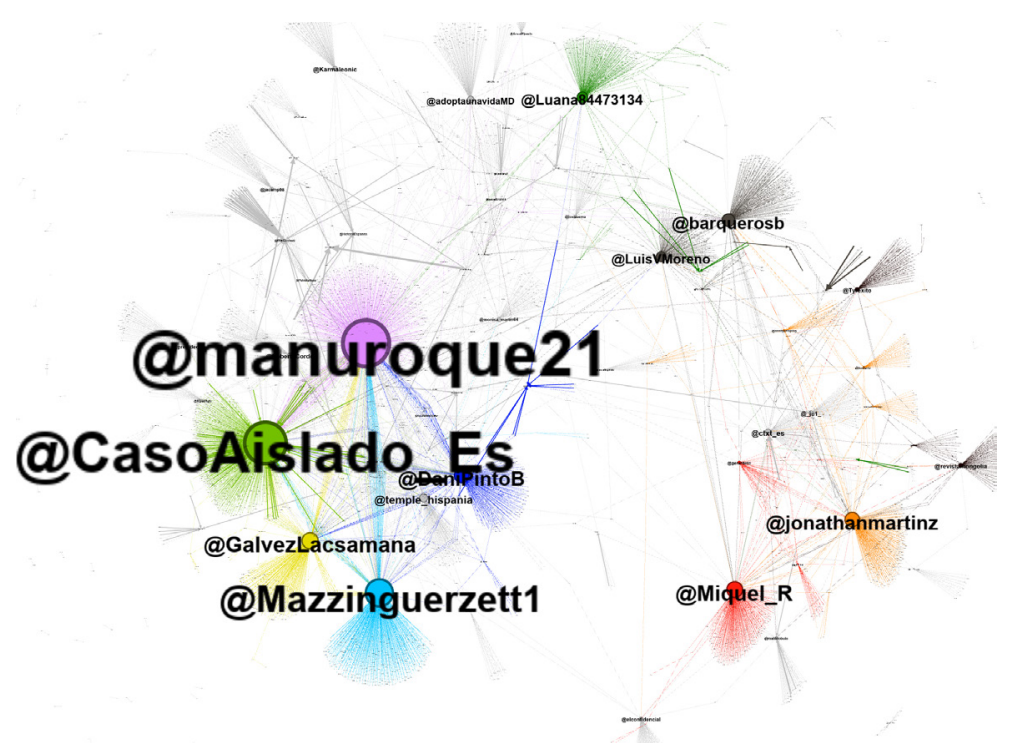

Figura 6: Análisis de redes de retuits, con los usuarios determinados por orden de eigenvector para ver los más influyentes. Fuente: Elaboración propia.

Aunque se obtuvieron 134 grupos de usuarios, solo algunos alcanzaron porcentajes reseñables y cuentan con influencers (Tabla 1). A través de un análisis de separación y clasificación de los grupos mediante los algoritmos OpenOrd y Yifan Hu sucesivamente, se determinó por eigenvector con un mayor tamaño de círculos quiénes eran los usuarios creadores de tendencia. Según se observa en la Figura 6, en la mayoría de los grupos hay un único influencer y no existe diversidad de actores salvo en los grupos animalistas y de la izquierda política. Los diez principales grupos representan el 74,31\% del tráfico total de tuits. La centralidad, aplicando un algoritmo de cálculo de centralidad del discurso de la red o eigenvector, se extiende alrededor de@manuroque21, mientras que los principales grupos que denuncian que la noticia es falsa quedan alejados de la centralidad de la red de tuits creados.

\begin{tabular}{|l|l|l|l|l|}
\hline Grupo-color & \multicolumn{1}{|c|}{ Influencer } & $\begin{array}{c}\text { Porcentaje de } \\
\text { tráfico generado }\end{array}$ & $\begin{array}{c}\text { Eigenvector } \\
\text { centralidad }\end{array}$ & Pagerank \\
\hline 1. Rosa & @manuroque21 & $12,14 \%$ & 1,000 & 0,061 \\
\hline 2. Verde & @CasoAislado_Es & $10,96 \%$ & 0,896 & 0,057 \\
\hline 3. Azul & @Mazzingerzzett1 & $7,95 \%$ & 0,585 & 0,037 \\
\hline 4. Marrón & @barquerosb & $7,24 \%$ & 0,270 & 0,017 \\
& @LuisVMoreno & 0,208 & 0,013 \\
\hline 5. Naranja & @jonathanmartinz & $6,35 \%$ & 0,308 & 0,020 \\
\hline 6. Rojo & @Miquel_R & $6,12 \%$ & 0,336 & 0,023 \\
\hline 7. Amarillo & @GalvezLacsamana & $4,63 \%$ & 0,343 & 0,020 \\
\hline 8. Morado & @DaniPintoB & $4,57 \%$ & 0,311 & 0,018 \\
\hline
\end{tabular}




\begin{tabular}{|l|l|l|l|l|}
\hline 9. Verde oscuro & @Luana84473134 & $3,98 \%$ & 0,225 & 0,015 \\
\hline $\begin{array}{l}\text { 10. Marrón } \\
\text { oscuro }\end{array}$ & $\begin{array}{l}\text { @revistamongolia } \\
\text { @Tyrexito }\end{array}$ & $3,13 \%$ & $\begin{array}{l}0,091 \\
0,088\end{array}$ & 0,006 \\
0,006
\end{tabular}

Tabla 1: Comunidades más importantes de opinión sobre Curro.

Fuente: Elaboración propia.

Como puede apreciarse en la Figura 6, existen seis grupos muy relacionados entre sí compartiendo muchas conexiones entre usuarios y prácticamente desvinculados de los otros, con excepción del Grupo 9 organizado en torno a una cuenta de contenido animalista con muchos seguidores y muy conectada con@CasoAislado_Es. La peculiar organización de nodos apunta a una red organizada alrededor de @-CasoAislado_Es formada por varios influencers que se activan en distintos periodos de tiempo, haciendo reaccionar a distintas cuentas de seguidores con las que comparten perfiles en común.

En la Figura 7, representado mediante el algoritmo Network Splitter $3 D$ en cuatro niveles de eje Z, se expresa la importancia según el algoritmo Pagerank (Brin, Page, 1998), estableciendo por alturas (Eje Z) a los usuarios a los que es más probable llegar, según una probabilidad del $85 \%$, sin conocer previamente la información. De esta manera se puede contrastar que un usuario cualquiera que accediera a Twitter en el periodo analizado llegaría más probablemente a los discursos de @manurroque21 y @CasoAislado_Es y, en tercer lugar, a @Mazzingerzzett1, tal como se aprecia en la Tabla 1.

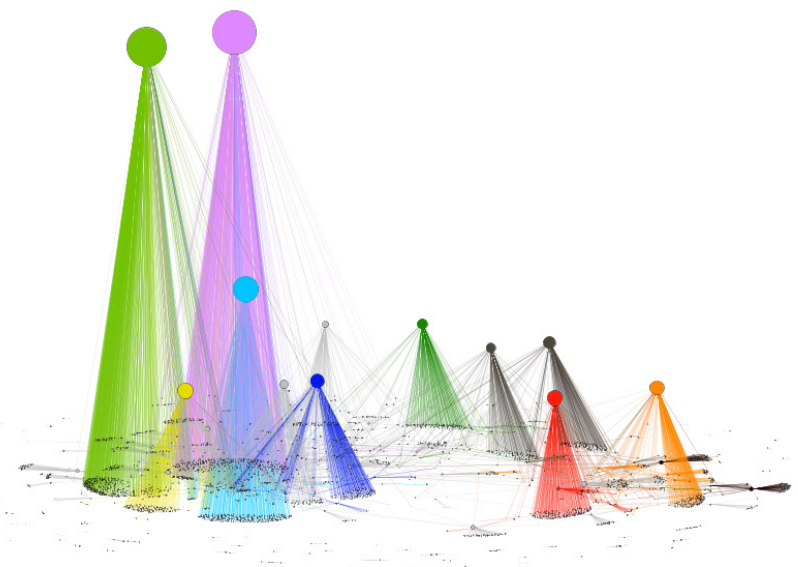

Figura 7: Determinación mediante Pagerank de los usuarios a los que es más probable llegar, con una fiabilidad del $85 \%$, desde un usuario cualquiera que no conozca la información. Fuente: Elaboración propia.

En la Figura 8 puede apreciarse mediante el algoritmo Network Splitter $3 D$ en cuatro niveles, dando peso al grado de salida en el gráfico y estableciendo por alturas en un eje Z, a los usuarios más redifusores de lo que escriben otros. Aunque el grado medio de difusión por cuenta es de 1,155, se puede comprobar que alrededor de la 
estructura de los influencers que difunden el bulo de Curro existen muchos usuarios redifusores. Un estudio más pormenorizado de los usuarios que retuitean arroja que provienen del ámbito animalista y muestra ejemplos de perfiles que llegan a redifundir mensajes hasta en 30 ocasiones. En el otro lado, se advierte que los principales autores de tuits o influencers no retuitean ningún contenido de otros usuarios.

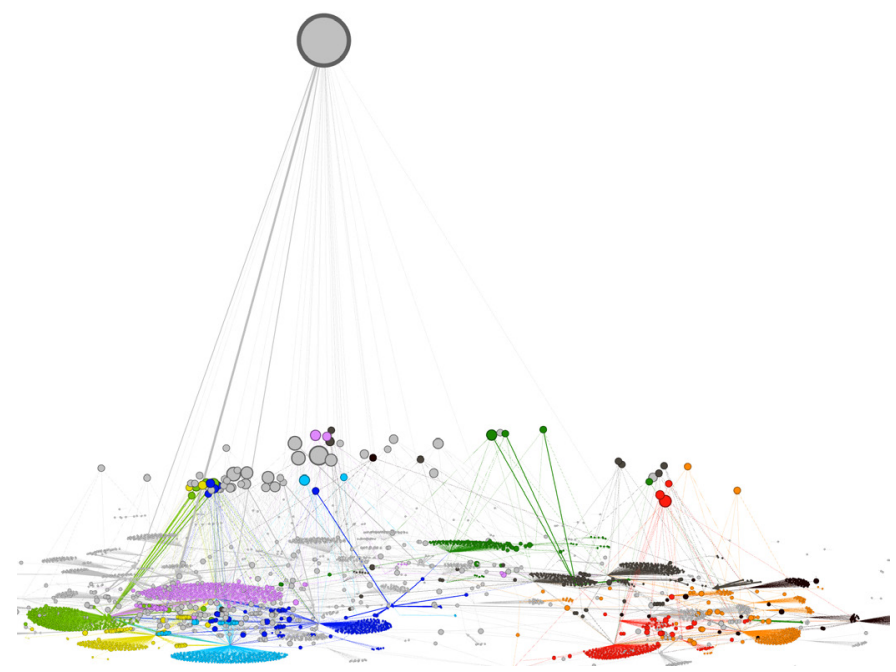

Figura 8: Análisis de redes de retuits, con los usuarios determinados por orden, tamaño y altura para ver aquellos que más redifunden. Fuente: Elaboración propia.

\subsection{Utilización de bots en la difusión}

Siguiendo los patrones que suelen identificar a las cuentas redirigidas por bots para crear y difundir mensajes de forma automática (Digital Forensic Research Lab, 2017), se han identificado tres tipos de perfiles:

- Cuentas de usuarios con nicks compuestos por un nombre de pila de seis o siete letras, seguido de una sucesión de entre siete y nueve números. Estas cuentas solo realizan retuits o menciones directas hacia otros perfiles del mismo formato. Se identifican retuits sobre fútbol, situación política en Venezuela, animalismo, política española, noticias sensacionalistas de las webs del presente estudio y Vox.

- Cuentas de usuarios cuyo nick comienza con cuatro o cinco letras al azar seguido de cuatro números. Solo retuitean o envían menciones a cuentas del mismo formato.

- Cuentas de usuarios cuyos nicks son una sucesión de letras y números al azar. Igualmente, solo retuitean o envían menciones a otras cuentas de su mismo formato.

Siguiendo estos patrones, se identifican 692 cuentas sospechosas de ser bots responsables de un total de 1.149 tuits $(12,60 \%$ de los tuits alrededor de Curro en el periodo analizado). Entre los usuarios influencers se determinan 140 posibles bots alrededor de @manurroque21, seguido, en orden decreciente por @CasoAislado_Es (72 bots), @Mazzingerzzett1 (57 bots),@GalvezLacsamana (43 bots), @DaniPintoB (36 bots), @Luana84473134 (35 bots) o @barquerosb (33 bots). 
Cabe destacar que una cuenta de estas características -@Luana84473134- se sitúa entre los 10 mayores influencers de este bulo, generando más del 3\% de tráfico de tuits totales. Igualmente se observan retuits desde cuentas sospechosas a los 22 minutos del primer tuit de @CarlosAsuajeS y a los 20 minutos de @CasoAislado_Es.

\subsection{Análisis de sentimientos}

El análisis de emociones, mostrado en la Figura 9, arroja que el sentimiento más extendido entre los tuits sobre Curro es el miedo, con una distancia de casi el doble con respecto a la siguiente emoción detectada, el enfado, a la que siguen la anticipación o razonamiento elaborado demostrando así que los mensajes recogidos parten de un discurso meditado cuya reacción no es instantánea. El disgusto, la tristeza y la confianza, por su parte, son mucho más escasos mientras que la alegría y la sorpresa resultan casi inexistentes.

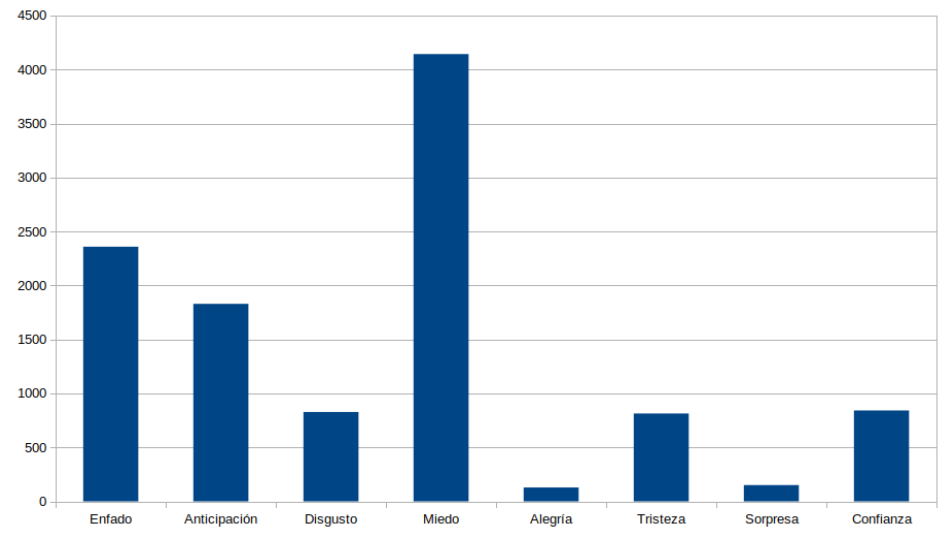

Figura 9: Análisis de sentimientos en los tuits. Fuente: Elaboración propia.

\subsection{Minería de texto de los discursos vertidos}

El estudio por minería de texto ofrece diversos datos como los 15 usuarios más referenciados (entre paréntesis el número de menciones): @manurroque21 (914), @ casoaislado_es (872),@mazzingerzett1 (534),@elconfidencial (348),@galvezlacsamana (314), @miquel_r (308),@danipintob (284),@jonathanmartinz (282),@ barquerosb (247),@luana84473134 (206),@luivmoreno (191),@temple_hispania (157),@adoptaunavidamd (128),@ctxt_es (106) y@karmaleonic (86). Los hashtags más empleados fueron \#curroestaenmicasa (134), \#salvaracurro (60), \#justiciaparacurro (6).

El estudio por escalamiento bidimensional para la determinación de los temas se realiza mediante el algoritmo Snowball y posterior ejecución de la metodología Kruskal y aplicación de distancia de Jaccard para representar los ocho principales cluster sobre las 160 palabras más empleadas (Figura 10). 


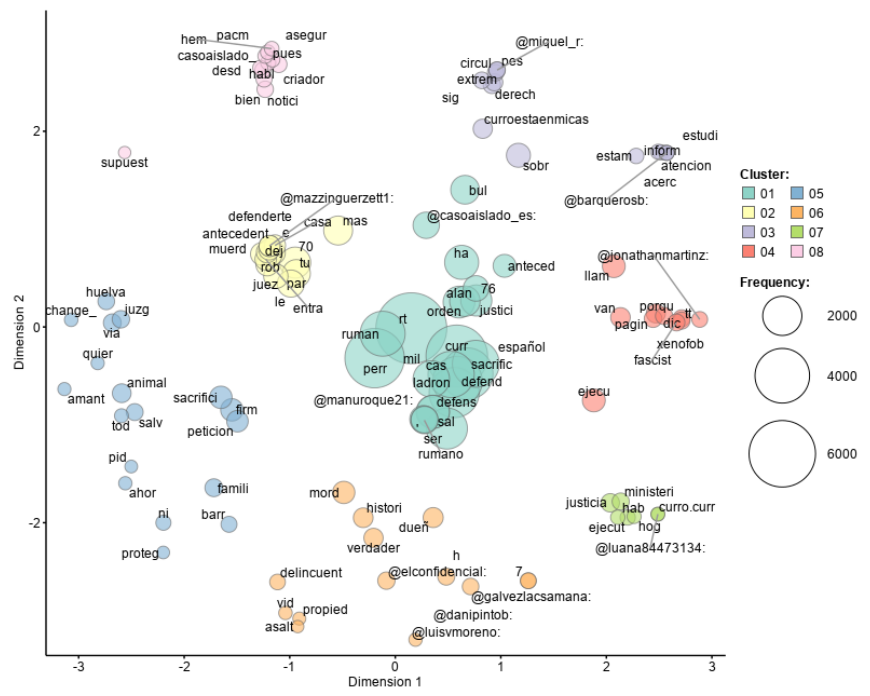

Figura 10: Escalamiento bidimensional sobre los textos de los tuits.

Fuente: Elaboración propia.

Se puede apreciar un elemento central de opinión con las palabras que aparecen con mayor frecuencia:

- Cluster 1 (verde): que expone la información de @casoaislado_es sobre la orden de justicia de sacrificio de Curro, tras defender su casa de un ladrón rumano.

- Cluster 2 (amarillo): ofrece el mismo perfil de comentarios que el Cluster 1 pero sin citarla fuente @casoaislado_es.

- Cluster 3 (morado): alerta del presidente de PACMA sobre la falsedad del caso.

- Cluster 4 (rojo): acusaciones de xenofobia y fascismo.

- Cluster 5 (azul): difusión de la petición en Change.org para salvar a Curro.

- Cluster 6 (naranja): difusión del desmentido por parte de El Confidencial.

- Cluster 7 (verde claro): petición al Ministerio de Justicia para evitar la ejecución de Curro.

- Cluster 8 (rosa): réplica de @ casoaislado_es ante las primeras acusaciones de falsedad por parte de PACMA.

\section{Conclusiones}

El caso de Curro, el perro "condenado", es un ejemplo claro de difusión intencionada de una noticia fabricada con amplia repercusión en el ecosistema social -el tema llegó a ser trending topic en Twitter en España durante los días 24 y 25 de diciembre-. Su emisión implicó a varios portales conocidos por la difusión de bulos y noticias sensacionalistas que, durante varios días, lanzaron de manera sucesiva, y presumiblemente coordinada, la información para conseguir aumentar la caja de resonancia de la noticia y, de esa manera, previsiblemente, aumentar el tráfico a sus sitios web. Esta práctica, vinculada a las formas de monetización de la publicidad programática, ha sido ampliamente identificada en otros procesos de desinformación similares. 
En la viralización de la noticia es posible identificar tanto perfiles de personas reales como usuarios que pudieran ser identificables como bots. Es básico para esta identificación no solo atender a la particular actividad de estos perfiles en la distribución de información (retuiteando y no creando contenido propio o, incluso, repitiendo varias veces el mismo contenido) o a la existencia de pautas en los nombres de estos perfiles (combinaciones de letras y números), sino también a la existencia de estructuras de comportamiento digital que pudieran estar organizadas para la redifusión de la información en intervalos periódicos. Según este estudio, el uso de este tipo de perfiles podría estar por encima del $10 \%$ del tráfico de tuits sobre Curro.

El componente humano -por lo general unido a perfiles activistas o políticos que se mueven entre el animalismo y la extrema derecha- contribuye a la estrategia de diseminación de la información ahondando en la activación de contenido en los momentos calientes de la viralización de la noticia. Prueba de ello es que, durante los dos días más intensos, dos particulares organizan sendas campañas de recogidas de firmas a través de Change.org.

Al hilo de las investigaciones citadas en este trabajo, parece claro que, para la activación de este componente humano, los contenidos de la noticia fabricada han de encajar con una narrativa ideológica preexistente. Este componente es visible no solo al analizar los temas de los que habitualmente hablan los perfiles implicados (Venezuela, Fútbol, Animalismo, Vox...) sino también al analizar las emociones con el miedo como sentimiento predominante. Así, aunque evidentemente no todos los usuarios reales que participan en la viralización coincidían con el ideario aquí resumido, sí podían identificarse con al menos uno de los mensajes del framing: la defensa a ultranza de los derechos de los animales y/o la veracidad otorgada a que un perro muerda a un ladrón y que este sea rumano. Esto podría indicar que los contenidos para crear esta noticia falsa han sido intencionadamente elegidos según estos marcos preexistentes y que es valiéndose de estos prejuicios previos como se seleccionan para su mención algunos de los perfiles que, ulteriormente, ayudan en la viralización. Este es el caso del perfil oficial del partido animalista PACMA.

Este componente ideológico y emocional es fundamental para entender por qué los canales de verificación que denuncian la falsedad de la noticia no llegan a lograr el alcance de la mentira. A pesar de las rectificaciones de periodistas y del propio PACMA, los avisos que advierten de la inexistencia de Curro -que nunca alcanzan la misma repercusión- conviven durante días con los tuits originales. Además, los contenidos sobre el caso solo desaparecen cuando dejan de ser reactivados por perfiles influencers coincidiendo con los desmentidos de El Confidencial y Maldito Bulo. Con todo, el análisis de probabilidad muestra que existió una mayor tendencia a difundir los tuits originales. Esto sugiere que un usuario ajeno al tema hubiera llegado mucho más fácilmente a la noticia falsa que a su rectificación.

El modus operandi descrito en este estudio podría encajar, por tanto, con una estrategia de diseminación de noticias falsas con fines predominantemente comerciales aunque apoyados sobre sustratos ideológicos. Favorece este hecho la existencia de un panorama de medios on line creados ex profeso para la publicación y posterior viralización de contenidos sensacionalistas, fake news incluidas, que emulan el diseño y la organización de los legítimos sitios de noticias, aprovechando para ello el consumo fragmentado e intermediado de información que tiene a las redes sociales como fuente fundamental. 


\section{Bibliografía}

AAVV (2017). El libro blanco de la información. Madrid: Asociación de Medios de Información.

Arce, S.; Menéndez, M. I. (2018). “Aplicaciones de la estadística al framing y la minería de texto en estudios de comunicación”. En Información, Cultura y Sociedad, 39, pp. 61-70. Disponible en: http://ppct.caicyt.gov.ar/index.php/ics/article/view/14459/45454575759151

Albright, J. (2016). “The \#Election 2016 Micro-Propaganda Machine”. Medium. Disponible: en: https://medium.com/@d1gi/the-election2016-micro-propaganda-machine-383449cc$1 \mathrm{fba}$

Allcott, H.; Gentzkow, M. (2017). "Social Media and Fake News in the 2016 Election". Journal of Economic Perspectives, Volume 31, Number 2, Spring 2017, pp. 211-236

Bakir, V.; Mcstay, A. (2017). "Fake News and The Economy of Emotions". Digital Journalism, 6(2), pp. 154-175.

Bastian, M., Heymann, S.; Jacomy, M. (2009). "Gephi: An Open Source Software for Exploring and Manipulating Networks". En Proceedings of the Third International ICWSM Conference (San Jose, California, 17-20 mayo de 2009). San Jose: Conferencia Internacional AAAI sobre Web y Redes Sociales.

Bell, E. J.; Owen, T.; Brown, P.d.; Hauka, C.; Rashidian, N. (2017). “The Platform Press: How Silicon Valley Reengineered Journalism”. En Tow Center, Columbia Journalism School.

Blondel, V.d.; Guillaume, J-L.; Lambiotte, R.; Lefebvre, E. (2008). "Fast unfolding of communities in large networks". Journal of Statistical Mechanics: Theory and Experiment 2008 (10), 1000.

Brin, S.; Page, L. (1998). "The Anatomy of a Large-Scale Hypertextual Web Search Engine". En Proceedings of the seventh International Conference on the World Wide Web (WWW1998). p: 107-117.

Digital Forensic Research Lab (2017): “\#BotSpot: Twelve Ways to Spot a Bot Some tricks to identify fake Twitter accounts”. Medium.com. Disponible en: https://medium.com/dfrlab/ botspot-twelve-ways-to-spot-a-bot-aedc7d9c110c

Castillo, T. (2017). "El 56,8 \% de los lectores españoles de prensa se informa a través de redes sociales, aunque sólo lee titulares y alguna noticia", Genbeta. Disponible en: https:// www.genbeta.com/redes-sociales-y-comunidades/el-56-8-de-los-lectores-espanoles-deprensa-se-informa-a-traves-de-redes-sociales-aunque-solo-lee-titulares-y-alguna-noticia

Coddington, M.; Molyneux, L.; Lawrence, R. (2014). "Fact Checking the Campaign", The International Journal of Press/Politics, 19.

Fernández Vallejo, A.m. (2018). "Comunicar emociones en el discurso metapolítico de twitter: el caso de \#MADURO versus @NICOLASMADURO”. En Observatorio (OBS*) Journal, pp. 175-194. Disponible en: http://obs.obercom.pt/index.php/obs/article/view/1214

Fernández-García, N. (2017). "Fake news: una oportunidad para la alfabetización mediática”. En Nueva Sociedad, no 269, mayo-junio de 2017, pp. 66-77.

Flanagin, A. J.; Metzger, M. J. (2007). "The role of Site Features, User Attributes, and Information Verification Behaviors on the Perceived Credibility of Web-based Information". En New Media \& Society I, pp. 319-342.

Gottfried J.; Shearer E. (2016). News use across social media platforms 2016. Pew Research Center. Disponible en: http://www. journalism.org/2016/05/26/news-use-across-socialmedia-platforms-2016/

Herman, E. S.; Chomsky, N. (2002). Manufacturing consent: The political economy of the mass media. New York: Knopf Doubleday Publishing Group. 
Hermida, A. (2011). 'Fluid Spaces, Fluid Journalism: The Role of The 'Active Recipient' in Participatory Journalism”. En Participatory Journalism: Guarding Open Gates at Online Newspapers, edited by Singer, J.; Hermida, A.; Domingo, D.; Heinonen, A.; Paulussen, S.; Quandt, T; Reich, Z.; Vujnovic, M, pp. 177-191. Malden: MA, John Wiley \& Sons.

Iyengar, S.; Hahn K. S. (2009). "Red media, blue media: evidence of ideological selectivity in media use". En Journal of Communication 59(1), pp. 19-39

Jewitt, R. (2009). "The Trouble With Twittering: Integrating Social Media into Mainstream News”. En International Journal of Media \& Cultural Politics 5 (3), pp. 233-246.

Kang, H.; Bae, K.; Zhang S.; Sundar, S. S. (2011). "Source Cues in Online News: Is The Proximate Source More Powerful than Distal Sources?”. En Journalism \& Mass Communication Quarterly 88 (4), pp. 719-736.

Kershner, J. W. (2005). The Elements of News Writing. Boston: MA, Pearson Allyn and Bacon.

Kovach, B.; Rosenstiel, T. (2007). The Elements of Journalism: What Newspeople Should Know and The Public Should Expect. New York: Three Rivers Press.

Lokot, T.; Diakopoulos, N. (2016). "News Bots: Automating News and Information Dissemination on Twitter”. Digital Journalism 4 (6), pp. 682-699.

Magallón, R. (2018). "La biblioteca digital sobre Donald Trump. Fact-checking frente a fake news". Estudios sobre el Mensaje Periodístico. V. 24, n. 1, (2018), pp. 273-282.

Mohammad, S.; Turney, P. (2010). "Emotions Evoked by Common Words and Phrases: Using Mechanical Turk to Create an Emotion Lexicon". En Proceedings of the NAACL-HLT 2010 Workshop on Computational Approaches to Analysis and Generation of Emotion in Text (Los Angeles, junio 2010). Los Angeles, California: North American Chapter of the Association for Computational Linguistics.

Nielsen, R. K.; Ganter S. A. (2017). "Dealing with Digital Intermediaries: A Case Study of the Relations between Publishers and Platforms". En New Media \& Society, April.

Peinado, F. (2018). "El negocio de la manipulación digital en España". El País, 23 de mayo de 2018. Disponible en: https://elpais.com/politica/2018/05/17/actualidad/1526571491_535772.html

Peterson, T. (2018). "The New York Times has folded its programmatic sales team into its larger ad sales org”, Digital Day. Disponible en: https://digiday.com/media/new-yorktimes-folded-programmatic-sales-team-larger-ad-sales-org/

Plutchik, R. (1980). “A general psychoevolutionary theory of emotion”. En Emotion: Theory, Research, and Experience, 1(3), pp. 3-33.

Ramírez, V.; Castellón, J. (2018). “'Caso Aislado', el fabricante español de 'fake news' vinculado a VOX”. La Sexta. Disponible en: https://www.lasexta.com/noticias/nacional/ asi-trabajan-caso-aislado-fabricante-espanol-fake-news-vinculado-vox_201810095bbcb81e0cf22dcc97718ef6.html

Sautera, D. A.; Eisner, F.; Ekman, P.; Scott S.k. (2010). "Cross-cultural recognition of basic emotions through nonverbal emotional vocalizations". En Proceedings of the National Academy of Sciences, 107 (6), pp. 2408-2412.

Silverman, C. (2016). "Here are 50 of the Biggest Fake News Hits on Facebook from 2016". BuzzFeed. Disponible en: https://www.buzzfeed.com/craigsilverman/top-fake-newsof-2016/

Shoemaker, P. J.; Reese, S. D. (2013). Mediating the Message in The 21st Century: A Media Sociology Perspective, 3rd ed. New York: Routeledge. 
Sundar, S. S. (2008). "The MAIN Model: A Heuristic Approach to Understanding Technology Effects on Credibility". En Digital Media, Youth, and Credibility, editado por Metzger M. J.; Flanagin, A. J, pp. 73-100. Cambridge, MA: The MIT Press.

Tandoc, E. C.; Lim, Z. W.; Ling, R. (2017). "Defining “Fake News"”. Digital Journalism, 6(2), pp. 137-153.

Tuchman, G. (1978). Making News: A Study in The Construction of Reality, New York: Free Press.

Ward, J-H. (1963). "Hierarchical grouping to optimize an objective function". En Journal of the American Statistical Association. 58, pp. 236-244.

Wardle, C. (2017). "Fake News. It's Complicated", First Draft News. Disponible en: https:// firstdraftnews.com:443/fakenews-complicated/ 